\title{
Névralgie trigéminale et anévrysme intra-caverneux de la carotide interne : à propos d'un cas
}

\section{Trigeminal neuralgia and aneurysm of the intracavernous internal carotid artery: a case report}

LAURENT GINESTE, CARLOS MADRID, BRUNO COURTOIS, DAMIEN DURAN*

\begin{abstract}
RÉSUMÉ
Le diagnostic différentiel entre névralgie trigéminale essentielle (NTE) et symptomatique est facilité lorsque le tableau clinique d'une NTE est constitué, et que l'examen clinique et neurologique est négatif. En revanche, ce diagnostic différentiel s'avère plus hasardeux lorsqu'une névralgie trigéminale symptomatique imite parfaitement les caractères cliniques d'une NTE. Cet article rapporte le cas d'une patiente de 81 ans avec des antécédents de dépression se plaignant d'une part, d'accès douloureux en décharge évoluant depuis les 3 dernières semaines et limités à une petite partie du territoire du nerf mandibulaire, et d'autre part de pertes de connaissances brèves dont la fréquence a augmenté récemment. Ce dernier point a conduit à pratiquer un examen tomodensitométrique qui a révélé la présence d'un anévrysme intracaverneux de la carotide interne. Les auteurs discutent les différentes hypothèses étiopathogéniques impliquant les conflits neurovasculaires dans le déclenchement des NTE, et soulignent l'importance de garder à l'esprit le fait qu'une cause tumorale cachée peut toujours être à l'origine d'une algie d'apparence essentielle. Cette observation d'une névralgie trigéminale en rapport avec un anévrysme intracaverneux de la carotide interne est la seule répertoriée à notre connaissance (Med Buccale Chir Buccale 2002; 8: 45-52).
\end{abstract}

mots clés : Névralgie trigéminale symptomatique, Névralgie trigéminale essentielle, Anévrysme, Sinus caverneux

médecine buccale chirurgie buccale VOL. $8, \mathrm{~N}^{\circ}$ 2002 page 45

\section{SUMMARY}

The differential diagnosis between so-called idiopathic trigeminal neuralgia and symptomatic trigeminal neuralgia is easy when the clinical criteria of tic douloureux are gathered and the neurologic examination is negative. Actually it is much more difficult, when a symptomatic neuralgia mimics the main features of an idiopathic trigeminal neuralgia. This paper reports the case of a women, aged 81 , with a history of depression and a complaint for painful epidodes during the last three weeks characterised by short-lasting attacks of intense, electric shocklike pain limited to the distribution of third division of the left trigeminal nerve and frequent fainting fits. This last criterion led us to ask for CT examination. CT images evidenced an aneurysm of the left intracavernous internal carotid artery probably related to the observed neuralgia. Authors discuss the etiopathogenic hypotheses reported by litterature about neurovascular conflicts and underline the necessity of keeping always in mind the possiblity of an hidden tumoral etiology to idiopathic trigeminal neuralgia-like syndroms. This case of trigeminal neuralgia related to an aneurysm of the left intracavernous internal carotid artery is to our knowledge the first reported (Med Buccale Chir Buccale 2002; 8: 45-52).

key words : Trigeminal neuralgia, Tic douloureux, Aneurysm, Cavernous sinus

\footnotetext{
* Equipe Médicale de Chirurgie Buccale, CHR de Toulouse France

Demande de tirés à part :

Laurent GINESTE UFR Odontologie 3 chemin des Maraîchers 31062 Toulouse Cedex
}

Article reçu le 8 février 2001. Accepté pour publication le 31 octobre 2001. 
médecine buccale chirurgie buccale

L. $8, \mathrm{~N}^{\circ} 1$

2002

page 46
Le statut de la névralgie trigéminale essentielle ou idiopathique s'est trouvé largement remis en cause au cours des 30 dernières années par l'amélioration des moyens d'imagerie [1] et les résultats des interventions neurochirurgicales [2] de la fosse cérébrale postérieure qui ont abouti à la résolution spectaculaire d'un grand nombre de conflits vasculo-nerveux. Cette résolution chirurgicale s'accompagne très régulièrement d'une amélioration, voire de la disparition totale des douleurs [3], qui font douter de l'opportunité de maintenir une séparation étanche entre névralgie «symptomatique» et névralgie dite essentielle parce qu'on n'a pu jusqu'ici identifier sa cause. On évalue aujourd'hui à $85 \%$ le nombre des patients atteints de névralgie trigéminale et présentant une compression du trijumeau dans la fosse cérébrale postérieure [4]. II s'agit le plus souvent d'une artère, plus rarement d'une veine et plus exceptionnellement encore d'une petite tumeur $[2,5,6]$. On a rapporté de longue date la possibilité pour une névralgie trigéminale symptomatique d'une tumeur de petite taille de mimer en tous points la sémiologie d'une névralgie essentielle [7]. Cette éventualité doit être constamment présente à l'esprit lors de l'établissement du diagnostic et du recours éventuel à une thérapeutique exclusivement médicale : quel que soit le caractère évocateur et presque pathognomonique d'une névralgie trigéminale, son caractère essentiel ne saurait être établi qu'après l'élimination par l'examen neurologique et d'éventuels examens complémentaires, en particulier d'imagerie, d'un conflit vasculaire et/ou tumoral.

Un cas de névralgie trigéminale symptomatique liée à un anévrysme du sinus caverneux et présentant la plupart des caractères cliniques d'une névralgie essentielle du trijumeau est rapporté.

\section{OBSERVATION}

En mai 1999, une patiente âgée de 81 ans, totalement édentée consulte pour des douleurs mandibulaires localisées au corps mandibulaire gauche. L'anamnèse rapporte des épisodes dépressifs anciens bien stabilisés par un traitement asso- ciant une benzodiazépine (Seresta $\left.{ }^{\circledR}\right)$, un neuroleptique antipsychotique (Largactil $\circledast$ ), et un barbiturique (Aparoxal $\circledast$ ).

L'interrogatoire révèle des douleurs particulièrement violentes, qui persistent depuis trois semaines et qui se sont aggravées lors des derniers jours, provoquant des insomnies en dépit du traitement sédatif instauré de longue date. La patiente se plaint d'irradiations douloureuses en direction du pharynx. Elle signale également de fréquents évanouissements de courte durée. Le médecin généraliste, fréquemment consulté ces derniers jours, a prescrit différents traitements antibiotiques qui n'ont apporté aucune amélioration. La patiente et sa famille sont persuadés qu'«on a oublié une dent».

L'examen exo buccal permet de retrouver des adénopathies sous mandibulaires bilatérales de caractère non spécifique. L'exploration du cou met en évidence une masse latéro-cervicale thyroïdienne particulièrement étendue du coté droit, occupant un espace triangulaire à sommet caudal entre le bord ventral du muscle sterno-cléidomastoïdien droit, le bord caudal droit du cartilage thyroïde, la ligne médiane et le bord crânial du ventre antérieur du muscle omo-hyoïdien droit.

L'examen endobuccal met en évidence dans une bouche totalement édentée, une crête mandibulaire gauche ne présentant aucune lésion muqueuse ou osseuse, visible ou palpable. On note une candidose buccale importante, en particulier sur la face dorsale de la langue mais sans douleur linguale associée.

La palpation de la zone désignée par la patiente (crête osseuse édentée du secteur 35-36) déclenche une douleur de type paroxystique accompagnée par un réflexe de protection de la patiente pendant environ 90 secondes. La répétition immédiate de la palpation ne provoque aucune douleur. II faut attendre une bonne quinzaine de minutes avant d'observer à nouveau une douleur provoquée à partir de la zone gâchette. Le reste de l'exploration buccale est négatif.

L'examen clinique est poursuivi par l'exploration de l'ensemble des paires crâniennes. Cet examen neurologique est totalement négatif.

L'examen orthopantomographique des maxillaires (Fig. 1) montre l'absence de reste radiculaire ou 

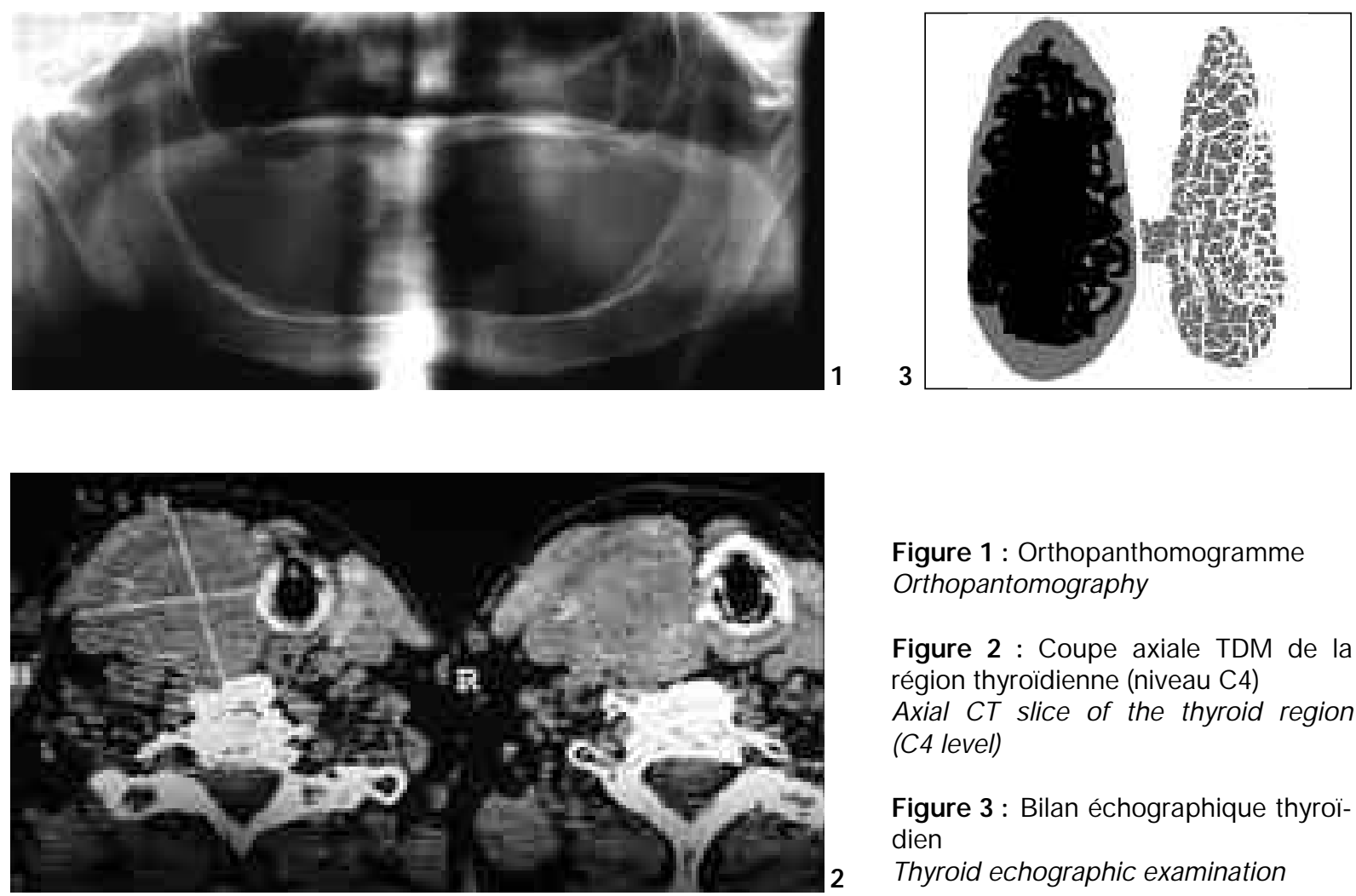

Figure 1 : Orthopanthomogramme Orthopantomography

Figure 2 : Coupe axiale TDM de la région thyroïdienne (niveau $\mathrm{C} 4$ )

Axial CT slice of the thyroid region (C4 level)

Figure 3 : Bilan échographique thyrö̈dien

Thyroid echographic examination

médecine buccale chirurgie buccale VOL. $8, \mathrm{~N}^{\circ}$ 2002 page 47

d'esquilles osseuses pouvant justifier ces douleurs. Le trajet du nerf alvéolaire inférieur est repéré à l'intérieur d'un canal mandibulaire de dimensions normales et de diamètre constant. Le foramen mentonnier droit est à distance de la crête osseuse, où la douleur semble se manifester avec le plus d'acuité. Aucun trait de fracture n'est repéré. La trabéculation osseuse est normale sans signe de remaniement inflammatoire pouvant évoquer un foyer ostéitique.

Un bilan biologique de routine est prescrit et ne retrouve guère qu'une élévation modérée des polynucléaires neutrophiles $\left(10,6 \times 10^{3} / \mathrm{mm}^{3}\right)$, une légère hyperglycémie à $1,30 \mathrm{~g} / \mathrm{l}(7,23 \mathrm{mmol} / \mathrm{l})$, une discrète hypercholestérolémie à $2,54 \mathrm{~g} / \mathrm{l}$ $(6,55 \mathrm{mmol} / \mathrm{l})$, et une élévation des transaminases (45 UI/I) et des Gamma GT (78 UI/I). Ce bilan est interprété comme proche de la normale compte tenu de son âge.

L'ensemble de ces explorations permet d'écarter une origine stomatologique des douleurs. Le diagnostic de névralgie trigéminale essentielle est alors évoqué en première intention. Néanmoins, la persistance des épisodes de perte de connaissance justifient une exploration complémentaire à la recherche d'une lésion éventuelle sur le trajet trigéminal mandibulaire extra crânien et intracrânien. Une exploration spécifique de la masse pharyngée est également demandée.

Un traitement antalgique de niveau II associant le paracétamol et un agoniste morphinique le dextropropoxyphène est prescrit (Propofan $\AA$, 3 comprimés par jour). Les antibiotiques jugés inutiles sont arrêtés et un traitement antifongique est mis en œuvre pour une durée de 15 jours.

L'examen tomodensitométrique de la thyroïde (Fig. 2) et l'examen échographique (Fig. 3) montrent la présence d'un volumineux nodule de $65 \times 65 \mathrm{~mm}$, tissulaire et vascularisé en périphérie, hypoéchogène et hétérogène au centre, probablement hémorragique ou nécrotique, de contours réguliers. L'examen conclut à un nodule tissulaire unique volumineux du lobe droit de la glande. 

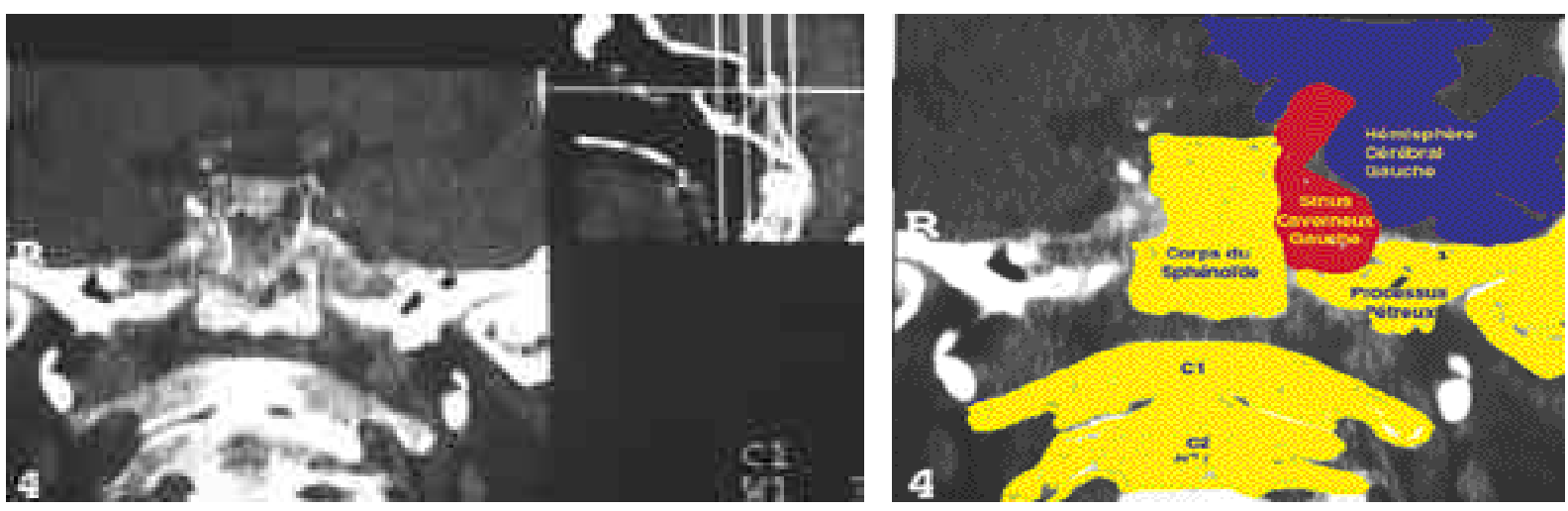

Figure 4 : Reconstruction bi-dimensionnelle coronale de la fosse cérébrale moyenne Coronal 2D reconstruction of the middle cerebral fossa

Figure 4bis : Schéma explicatif

médecine buccale chirurgie buccale L. $8, \mathrm{~N}^{\circ} 1$ 2002 page 48
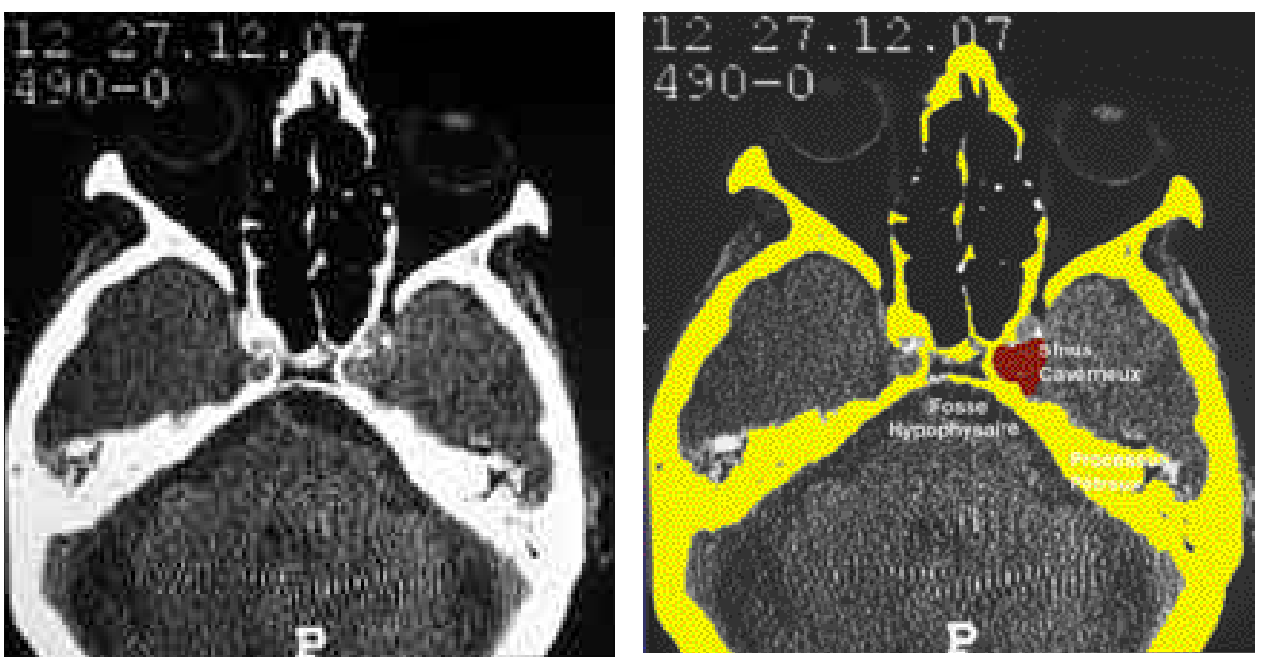

Figure 5 : Coupes axiales TDM de la fosse cérébrale moyenne

Axial CT slices of the middle cerebral fossa

Figure 5bis : Schéma explicatif
L'examen tomodensitométrique de la base du crâne et de la région temporale (Fig. 4 et 5) a été réalisé à l'aide de coupes d'acquisitions axiales et de reconstructions 2D multiplanaires sans injection de contraste étant donné l'âge de la patiente. L'exploration permet d'objectiver, à l'étage intracrânien, une convexité de la paroi du sinus caverneux du coté gauche avec une image d'encoche du bord latéral du corps du sphénoïde qui témoigne d'une malformation anévrysmale intracaverneuse probablement responsable de la névralgie trigéminale. Devant ce tableau, le diagnostic d'une névralgie trigéminale liée à une lésion intra-caverneuse est retenu.

Cette patiente est donc adressée dans le service de neurochirurgie. Le bilan de la consultation de neurochirurgie souligne le rôle probable d'une méga-dolicho-carotide gauche au niveau du sinus caverneux. Par ailleurs le kyste thyroïdien droit, initialement inflammatoire et douloureux est alors sans manifestation. L'examen neurologique ne retrouve pas de trouble sensitif évident et le réflexe cornéen est présent. Du fait de l'efficacité du traitement par le Propofan ${ }^{\circledR}$, et en raison de l'âge de la patiente et des associations médicamenteuses, le traitement par les anti-épileptiques de référence n'a pas été retenu, pas plus qu'un abord chirurgical particulièrement risqué.

La patiente relativement soulagée par le traitement antalgique per os a été placée sous la surveillance de son médecin traitant. 


\section{COMMENTAIRES}

La névralgie trigéminale est la plus fréquente des névralgies de nerfs crâniens. Son incidence annuelle a été évaluée à 4 pour 100000 habitants aux USA [8]. Cette névralgie est caractérisée par des crises douloureuses intenses qui intéressent le territoire d'une ou plusieurs branches du trijumeau.

Dans la classification des algies bucco-faciales, on distingue classiquement deux types de névralgies : la névralgie symptomatique, correspondant à une étiologie déterminée; et la névralgie essentielle ou idiopathique, d'étiologie inconnue. Ces deux types de névralgies s'opposent le plus souvent cliniquement point par point. En effet, les caractères de la douleur que sont sa qualité, sa durée, son rythme, son intensité, son déclenchement, et d'autre part le résultat de l'examen neurologique, permettent de distinguer ces deux entités (Tableau 1).

Dans l'observation rapportée, le caractère intense de la douleur, son déclenchement par la stimulation d'une zone gâchette, l'existence d'une période réfractaire et le résultat négatif de l'examen clinique et surtout neurologique, ont amené à poser le diagnostic provisoire de névralgie essentielle. Cependant il existe, de façon exceptionnelle, des névralgies symptomatiques mimant les caractères cliniques de la névralgie essentielle du trijumeau, notamment lors de certaines scléroses en plaques dont les lésions intéressent la racine descendante du trijumeau. II faut donc toujours garder à l'esprit que le diagnostic de névralgie essentielle doit rester un diagnostic d'élimination. De même, une algie essentielle peut être le siège révélateur d'une lésion compressive de la racine $d u$ trijumeau. Ces algies se produisent à un stade où le retentissement mécanique est trop discret pour entraîner un déficit décelable de la sensibilité et/ou les douleurs continues qui sont un signe plus fréquent des compressions progressives. II en résulte que devant toute névralgie trigéminale, un examen neurologique minutieux doit être conduit pour étudier la sensibilité du territoire douloureux et la sensibilité cornéenne. Cependant, les signes neurologiques ne sont présents que lorsque la lésion atteint l'intégrité du nerf sur son trajet et non pas en cas de lésion irritative périphérique. Dans le cas présenté, l'examen neurologique s'est révélé négatif. médecine buccale chirurgie buccale

VOL. $8, \mathrm{~N}^{\circ}$ 2002 page 49

Tableau 1 : Tableau clinique des névralgies trigéminales Clinical criterias of trigeminal neuralgia

\begin{tabular}{|c|c|c|}
\hline & Névralgie essentielle & Névralgie symptomatique \\
\hline Incidence féminine & Majoritaire & Variable \\
\hline Age de début & Après 50 ans & Variable \\
\hline Qualité & Fulgurante, en éclair & Douleurs lancinantes, irradiantes \\
\hline Durée & Brève & Prolongée \\
\hline Rythme & $\begin{array}{l}\text { Début et fin de crise brutal, } \\
\text { paroxystique }\end{array}$ & Fond continu avec paroxysmes \\
\hline Intensité & Atroce & Moyenne, modérée \\
\hline Déclenchement & $\begin{array}{c}\text { Stimulus de la zone gâchette } \\
\text { Période réfractaire }\end{array}$ & Douleurs continues \\
\hline Exemen neurobiologique & Négatif & Positif \\
\hline
\end{tabular}


Tableau 2 : Etiologies des névralgies trigéminales symptomatiques Etiology of symptomatic trigeminal neuralgia

\begin{tabular}{|c|c|}
\hline Siège & Etiologies \\
\hline Tronc cérébral & $\begin{array}{l}\text { Sclérose en plaque } \\
\text { Tumeur } \\
\text { Processus vasculaire }\end{array}$ \\
\hline Angle ponto-cérébelleux & $\begin{array}{l}\text { Neurinome du VIII } \\
\text { Autres tumeurs de voisinage } \\
\text { Anévrysmes artério-veineux } \\
\text { Lésions d'arachnoïdite }\end{array}$ \\
\hline Ganglion de Gasser & $\begin{array}{l}\text { Tumeurs du Gasser } \\
\text { Tumeurs de la base du crâne } \\
\text { Zona }\end{array}$ \\
\hline Loge caverneuse & $\begin{array}{l}\text { Tumeurs de l'orbite } \\
\text { Méningiome } \\
\text { Tumeur hypophysaire } \\
\text { Anévrysme de la carotide interne } \\
\text { Thrombose du sinus caverneux }\end{array}$ \\
\hline Base du crâne & $\begin{array}{l}\text { Tumeurs de la base du crâne } \\
\text { Fractures de la base du crâne }\end{array}$ \\
\hline Trajet exo crânien & $\begin{array}{l}\text { Fractures maxillaires ou mandibulaires } \\
\text { Infections } \\
\text { Tumeurs malignes }\end{array}$ \\
\hline
\end{tabular}

En fait, une névralgie trigéminale ne pourra être qualifiée d'essentielle, parfois de façon transitoire, qu'après le résultat négatif d'examens complémentaires, tomodensitométrie et surtout IRM [9] de la fosse cérébrale postérieure, à la recherche d'une étiologie symptomatique de la névralgie (Tableau 2).

Les trois étiologies principales retenues pour la névralgie trigéminale «essentielle» sont la compression nerveuse par une artère dans l'espace pré ponto-cérébelleux, la compression par une tumeur, et la sclérose en plaque.

Ces atteintes entraîneraient un processus de démyélinisation créant ainsi un «court-circuit» entre fibres de la douleur et fibres sensitives non nociceptives.

L'étiologie compressive a été largement discutée du fait des résultats contradictoires des dissections post-mortem (par ailleurs difficilement extrapolable à l'anatomie vasculaire sur le vivant) ainsi que de l'existence de contacts vasculaires asymptomatiques [10]. Cependant la décompression vasculaire chirurgicale entraîne dans la majorité des cas une rémission de la douleur qui plaide en faveur de cette étiopathogénie.

Différents auteurs $[9,1]$ ont mis en évidence selon des critères neuroradiologiques, par des techniques de tomographie par résonance magnétique tridimensionnelle, et par angiotomographie à haute définition par résonance magnétique, des corrélations entre l'intensité de la douleur et l'interaction neurovasculaire. Une image radiologique de compression ou de dislocation d'un nerf par une artère est toujours associée à des symptômes de névralgie trigéminale, alors qu'un simple contact entre un vaisseau et un nerf n'est pas spécifiquement responsable d'une symptomatologie. Dans ce cas, d'autres facteurs peuvent être impliqués. La compression vasculaire concerne généralement le dernier centimètre du trajet de la racine nerveuse avant sa pénétration dans le pont (Fig. 6). Lorsque la névralgie trigéminale 


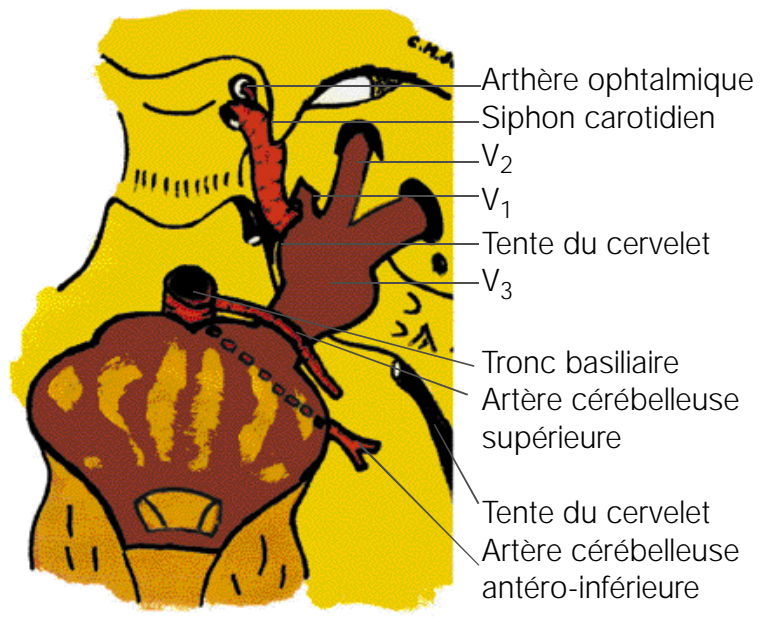

Figure 6 : Conflits artério-nerveux au niveau de la racine sensitive du $\mathrm{V}$

Arterio-nervous conflicts of the trigeminal sensitive root

concerne les $2^{\mathrm{e}}$ et $3^{\mathrm{e}}$ branches du nerf trijumeau, c'est généralement du à une compression de la partie rostrale antérieure de la racine sensitive par l'artère cérébelleuse supérieure. Lorsque $c^{\prime}$ est dans le territoire de la $1^{\text {re }}$ branche du trijumeau que se manifeste la névralgie, c'est généralement en rapport avec une compression de la portion caudale et postérieure de la racine par l'artère cérébelleuse antéro-inférieure [3].

Dans notre observation, l'examen tomodensitométrique met en évidence un anévrysme caverneux de la carotide interne.

La prévalence des anévrysmes intra-caverneux de la carotide interne est mal connue. Selon les auteurs, l'anévrysme de la portion intra-caverneuse de l'artère carotide interne représente entre 1,9 et $9 \%$ des anévrysmes intracrâniens. Par ailleurs, $14 \%$ des anévrysmes carotidiens internes seraient intra-caverneux [11]. Les facteurs de risque sont communs avec ceux des autres anévrysmes intracrâniens : âge avancé, sexe féminin et hypertension artérielle. Ces lésions ont en général une évolution spontanée bénigne, avec néanmoins de fréquents retentissements sur les nerfs crâniens de voisinage. Cependant cet anévrysme peut se compliquer de surinfections surtout à staphylocoques dorés [12] accompagnées de thromboses, d'hémorragies sub-arachnoïdiennes ou de fistules carotido-caverneuses.

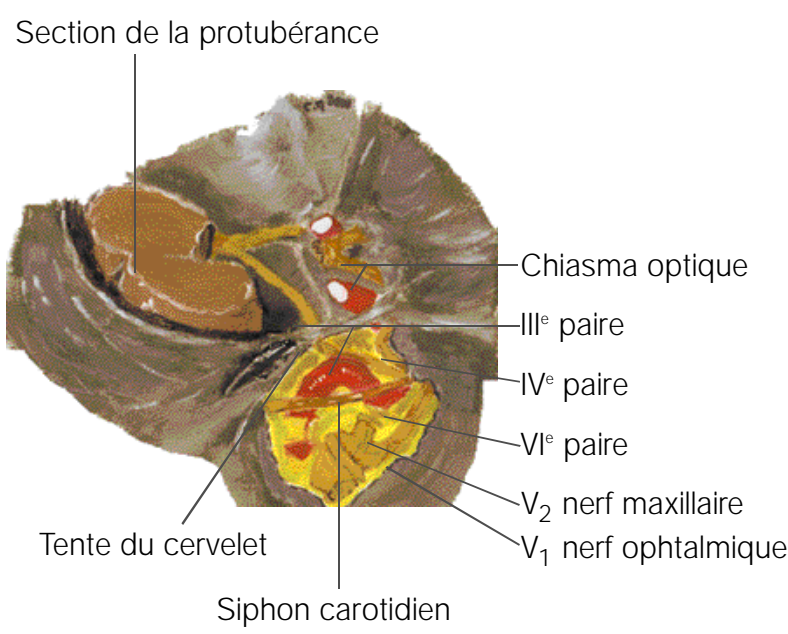

Figure 7 : Rapports nerveux de la carotide interne intera-caverneuse

Intra-cavernous segment of internal carotid artery

La disposition anatomique de la carotide interne à l'intérieur du sinus caverneux favorise probablement ces malformations vasculaires. La carotide forme un véritable siphon dès sa sortie de l'apex pétreux, par une courbure ascendante en haut en avant et en dedans, suivie par une courbure ascendante en haut en arrière et en dehors qui lui permet de rejoindre le bord antéro-latéral de la fosse hypophysaire, juste en dessous et en dedans du processus clinoïde antérieur. Outre les effets hémodynamiques évidents provoqués par la disposition en siphon, il faut noter que les branches de la carotide interne issues de sa portion post caverneuse (artères ophtalmiques ou branches du polygone de Willis) ont toutes un diamètre sensiblement inférieur au diamètre $d u$ tronc provoquant un effet d'entonnoir ce qui soumet celui-ci à des contraintes élastiques importantes. Les symptômes sont liés à la compression des éléments situés à proximité ou dans le sinus caverneux ou sur les lobes frontaux et temporaux adjacents. Une ou plusieurs des paires crâniennes situées dans ou au voisinage du sinus caverneux (Fig. 7) peuvent être atteintes par la compression qui peut donc conduire à une ophtalmoplégie (III, IV et VI) à une abolition du réflexe cornéen $\left(V_{1}\right)$ à une névralgie trigéminale $\left(V_{1}, V_{2}, V_{3}\right)$ comme dans notre observation, enfin à un hémi-spasme du médecine buccale chirurgie buccale VOL. $8, \mathrm{~N}^{\circ}$ 2002 page 51 
médecine buccale chirurgie buccale

L. $8, \mathrm{~N}^{\circ} 1$ 2002

page 52 facial (VII) [13], La III' paire, nerf oculomoteur, circule d'abord à l'intérieur du sinus caverneux puis pénètre à l'intérieur de sa paroi latérale où elle se divise avant de s'engager dans la fissure orbitaire supérieure. Le IV, nerf trochléaire, est presque constamment dans la paroi latérale, tandis que le VI, nerf abducens, traverse le sinus caverneux en circulant en dessous et légèrement latéralement à la carotide interne. Le $V_{1}$, nerf ophtalmique est haut situé dans la paroi latérale, à l'angle inféro-latéral du sinus, juste au-dessous de la Illème paire. II est pratiquement au contact de la carotide interne au sortir de l'apex pétreux. Le $V_{2}$ est au pied de la paroi antéro-latérale du sinus caverneux, légèrement étalée en dehors. Le $V_{3}$ est situé à distance dans l'épaisseur de la cavité trigéminale (cavum de Meckel) et son atteinte, lors d'un anévrysme intra-caverneux ne peut s'expliquer que par une dilatation carotidienne majeure comprimant la branche de division à travers la paroi latérale du sinus caverneux, la racine apparente pré-ganglionnaire du $\mathrm{V}$ (ancien plexus semi-lunaire) se trouvant trop loin de la carotide intra-caverneuse pour subir une compression pré-pontique.

Si le diagnostic différentiel entre névralgie trigéminale symptomatique et névralgie trigéminale essentielle est facilité lorsque la douleur s'entoure d'un riche cortège de signes neurologiques, il peut être difficile lorsque la névralgie simule une névralgie essentielle dans un tableau clinique discret.

Le diagnostic de névralgie essentielle reste un diagnostic d'élimination, et ne peut être avancé qu'après examen neurologique approfondi et négatif, et des examens d'imagerie négatifs, à la recherche d'une étiologie compressive tumorale ou vasculaire. L'observation d'un anévrysme intra-caverneux à l'origine d'une névralgie trigéminale d'allure essentielle est exceptionnelle : bien que cette possibilité soit rapportée par la littérature nous n'en avons pas retrouvé d'autre observation. Le cas que nous rapportons est à verser au débat controversé qui se développe autour des étiologies compressives qui ne sont toujours pas clairement identifiées comme un facteur étio-pathogénique exclusif.

\section{RÉFÉRENCES}

1 - Masur H, Papke K, B ongartz G, Vollbrecht K. The significance of three-dimensional MR-defined neurovascular compression for the pathogenesis of trigeminal neuralgia. J Neurol 1995; 242: 93-8.

2 - J ANNETTA PJ. Arterial compression of the trigeminal nerve at the pons in patients with trigeminal neuralgia. J Neurosurg 1967; 26: 159-62.

3 - J ANNETTA PJ. Microchirurgical approach to the trigeminal nerve for tic douloureux. Progress in neurosurgical. Surgery 1976; 7: 180-200.

4 - LOESER J D. Trigeminal neuralgia and atipical face pain (pp 699-709) Textbook of Pain. Ed Churchill Livingstone. 3 Edition. Chicago 1999.

5 - LAZAR ML, KiRKPATRICK J B. Trigeminal neuralgia and multiple sclerosis: Demonstration of the plaque in an operative case. Neurosurgery 1979; 5: 711-7.

6 - BURChiel KJ. Percutaneous retrogasserian glycerol rhizolysis in the management of trigeminal neuralgia. J Neurosurg 1988; 69: 361-6.

7 - Vaysse F, Madrid E, Campan P, Guichard M, Duran $D$. Approche clinique des douleurs oro-faciales chroniques. Med Buccale Chir Buccale 1998; 4: 25-36.

8 - Katusic S, Beard M, Bergstralh E, Kurland L. Incidence and clinical features of trigeminal neuralgia, Rochester, Minnesota, 1945-1984. Ann Neuro 1990; 27: 89-97.

9- Meaney J FM, Eldridge PR, Dunn lT, Nixon te, WhITEHOUSE GH, M ILES J B. Demonstration of neurovascular compression in trigeminal neuralgia with magnetic resonance imaging. J Neurosurg 1995;83: 799-805.

10- Hamlyn PJ, KIng TT. Neurovascular compression in trigeminal neuralgia: a clinical and anatomical study. J Neurosurg 1992; 76: 948-4.

11- Horowitz M, Fichtel F, Samson D, Purdy P. Intracavernous carotid artery aneurysms: The possible importance of angiographic dural waisting. A case report. Surg Neurol 1996; 46: 549-52.

12 - Destian S, Tung H, Gray R, Hinton DR, Day J, FUKUSHIMA T. Giant infections intra-cavernous carotid artery aneurysm presenting an intractable epistaxis. Surg Neurol 1994; 41: 472-6.

13- Ryu H, Yamamoto S, Sugiyama K, Uemura K, Miramoto T. Hemifacial spasm caused by vascular compression of the distal portion of the facial nerve. J Neurosurg 1998; 88: 605-9. 